\title{
Early changes in left ventricular myocardial function by 2D speckle tracking layer-specific technique in neonates with hyperbilirubinemia
}

\author{
Zimian Chen $^{1,2 \#}$, Suming Zhang ${ }^{1,2 \#}$, Aijuan Fang ${ }^{1,2} \wedge$, Jun Shao ${ }^{1,2}$, Hong Shen ${ }^{1,2}$, Bugao Sun ${ }^{1,2}$, \\ Guanjun Guo ${ }^{1,2}$, Lei Liu ${ }^{1,2 \wedge}$
}

${ }^{1}$ Department of Ultrasound Diagnosis, Affiliated Kunshan Hospital of Jiangsu University, Kunshan, China; ${ }^{2}$ Department of Ultrasound Diagnosis, Affiliated Drum Tower Hospital of Nanjing University, Nanjing, China

Contributions: (I) Conception and design: Z Chen, L Liu; (II) Administrative support: B Sun, G Guo; (III) Provision of study materials or patients: Z Chen, S Zhang, A Fang, J Shao, H Shen, G Guo, L Liu; (IV) Collection and assembly of data: Z Chen, S Zhang, A Fang, G Guo, L Liu; (V) Data analysis and interpretation: Z Chen, G Guo, L Liu; (VI) Manuscript writing: All authors; (VII) Final approval of manuscript: All authors.

\#These authors contributed equally to this work.

Correspondence to: Lei Liu; Guanjun Guo. Department of Ultrasound Diagnosis, Affiliated Drum Tower Hospital of Nanjing University, No.321 Zhongshan Street, Nanjing 210000, China. Email: 614052843@qq.com; snakeinsands@163.com.

Background: Hyperbilirubinemia $(\mathrm{HBN})$ can cause myocardial injury in neonates. Advancement in myocardial deformation imaging allows the detection of subclinical changes in myocardial contractility. The present study aimed to evaluate the changes in left ventricular contractility in newborns with hyperbilirubinemia by 2D speckle tracking imaging (STI).

Methods: A group of 134 neonates who reached the diagnostic level of HBN as the HBN group was selected. The control group included 56 healthy newborns. The interventricular septum, anterior partition, anterior wall, sidewall, posterior wall, and inferior wall were separated into the basal, middle, and apical segments. In each segment, speckle tracking analysis was performed in the subintimal, middle, and subadventitial myocardium. The overall longitudinal strain of the myocardium in different ventricular walls and segments and global longitudinal strain (GLS) were computed. At the same time, the laboratory results of blood gas analysis, blood routine tests, liver function, and myocardial enzyme spectrum in HBN neonates were collected and correlated with the left ventricular stratified strain parameters.

Results: The gradient of the left ventricular GLS had the same characteristics in both groups of newborns. There was a decreasing trend of longitudinal strain (LS) from the intima to the adventitia (i.e., GLSendo > GLSmid > GLSepi). This gradient was also present in stratified LS in each myocardial segment $(\mathrm{P}<0.001)$. The LS showed an increasing trend from the basal to the apical segment $(\mathrm{P}<0.001)$. The LS of the ventricular septum, anterior wall (or anterior septum), inferior wall, lateral wall, and posterior wall showed a decreasing trend $(\mathrm{P}<0.001)$. Stratified strain parameters of the ventricular wall (i.e., the 3-layer myocardium: LSendo-SEPT, LSmid-SEPT, and LSepi-SEPT) were all significantly lower in the HBN group than in the control group ( $\mathrm{P}=0.019, \mathrm{P}=0.019$, and $\mathrm{P}=0.023$, respectively). LSedo-ANT, LSmid-ANT, and LSepi-ANT were also reduced, and the difference between LSendo-ANT and LSepi-ANT was statistically significant. The segmental stratified strain parameters (i.e., the apical 3-layer myocardium: LSepi-a, LSmid-a, and

^ ORCID: Zimian Chen, 0000-0003-0891-4125; Lei Liu, 0000-0002-5416-0099; Suming Zhang, 0000-0002-7216-9898; Hong Shen, 0000-00028346-8201; Aijuan Fang, 0000-0002-1492-1287; Guanjun Guo, 0000-0002-0184-2010; Bugao Sun, 0000-0003-4969-8270. 
LSepi-a) decreased, and the difference in LSepi-a was statistically significant $(\mathrm{P}=0.043)$. Overall strain parameters (i.e., the 3-layer myocardial overall strain: GLSendo, GLSmid, and GLSepi) were reduced, but the difference was not statistically significant $(\mathrm{P}=0.612, \mathrm{P}=0.653$, and $\mathrm{P}=0.585$, respectively). The subclinical changes in systolic function in the HBN group, reflected by the parameters of longitudinal myocardial strain, correlate to some extent with multiple results of laboratory tests.

Conclusions: 2DSTI stratified strain technology can quantitively evaluate changes in the LS of the left ventricle in different ventricular walls, wall segments, and layers of the myocardium.

Keywords: Speckle tracking; hyperbilirubinemia; echocardiography; left ventricular systolic function; myocardial deformation; newborns

Submitted Feb 18, 2021. Accepted for publication Aug 10, 2021.

doi: 10.21037/qims-21-197

View this article at: https://dx.doi.org/10.21037/qims-21-197

\section{Introduction}

With the modification of the family planning policy and rapid development of perinatal medicine in China, hyperbilirubinemia $(\mathrm{HBN})$ among newborns has become increasingly common in clinical practice. At low concentrations, bilirubin acts as a scavenger of oxygen free radicals, and its antioxidant effects prevent the damage of cells by superoxide compounds. Therefore, the physiological concentration of bilirubin has a protective effect on the organism, most of which can disappear naturally without leaving any sequelae (1). In some newborns, the bilirubin level continues to increase and cannot subside on its own, resulting in HBN of the newborn. The main clinical focus has generally been on the damage of bilirubin to the central nervous system and the induction of bilirubin encephalopathy $(2,3)$. In-depth research has revealed that HBN affects myocardial contractility, and this effect of bilirubin increases attention (4).

Echocardiography is essential for evaluating local and overall myocardial function. Echocardiography offers several advantages; it is non-invasive, does not involve radiation, and is simple and easy to operate. Therefore, this technique is the first choice for imaging modalities in diagnosing heart disease in both adults and neonates. 2D speckle tracking imaging (STI) is a recently developed method to evaluate local and global myocardial function based on strain and strain rate imaging technology. 2DSTI can diagnose earlier than the commonly used measurement of left ventricular ejection fraction (LVEF) (5). Longitudinal peak strain refers to the shortening activity of each ventricular wall segment along the longitudinal direction of heart fibers. The core content of the myocardial band theory states that the movement of the myocardium along the long axis of the heart occurs mostly during the left ventricle contraction, accounting for about $70 \%$ of the overall movement of the myocardium $(6,7)$. Compared with the traditional index of overall pump function, longitudinal strain (LS) can more sensitively reflect the degree of myocardial damage, which may decrease before changes in LVEF take place, is more reproducible than radial strain and circumferential strain, at the same time, the sensitivity of LS is also better $(8,9)$. The early speckle tracking technology only analyzed the left ventricular wall as a whole but did not discriminate between the 3 layers of myocardial fibers of which the ventricular wall is composed, that is, the inner, middle, and outer layers of the myocardium. The subintimal and subpericardial myocardium contraction can shorten the ventricle, while the contraction of the middle myocardium can shrink the ventricular cavity (10). Due to the unique arrangement of myocardial fibers and the synergy between the different layers of the myocardium, different pathological factors have distinct effects on the function of each layer of the ventricular wall. Local or single-layer myocardial injury is often difficult to detect, particularly at an early stage. It may be more meaningful to study the 3 layers of the myocardium separately for the evaluation, treatment, and prognosis of myocardial damage. Layered strain technology divides the left ventricular wall myocardium into 3 layers based on high frame rate $2 \mathrm{D}$ ultrasound images. The technology of best pattern matching is used to track frame by frame the displacement of each layer of myocardial acoustic spots in the image during the cardiac cycle. The trajectory of this movement is used to quantitate the longitudinal and circumferential strain in different segments 
of the left ventricle and the overall myocardium. This approach enables the evaluation of the exercise capacity of fibers in each layer, locally and in the entire myocardium, and can detect limited myocardial injury in the early stage of the disease (11). The present study used the 2DSTI stratified strain technique to assess the characteristics of the LS of the 3layers of the left ventricular wall and the changes in systolic function in neonates with $\mathrm{HBN}$, to provide a more sensitive and efficient screening method for detecting subclinical local and overall myocardial damage.

\section{Methods}

\section{Patients}

We selected 190 full-term newborns hospitalized or treated as outpatients at the Department of Pediatrics and Obstetrics of the Kunshan Hospital Affiliated to Jiangsu University from December 2018 to June 2020. There were 99 boys and 91 girls. The newborns were divided into the HBN group and control group, according to the diagnostic criteria for HBN. The HBN group included 74 males and 60 females with an average age of $6.25 \pm 3.79$ days and average gestational age of $271.60 \pm 8.39$ days. The control group included 25 males and 31 females with an average age of $5.36 \pm 3.09$ days and average gestational age of $272.95 \pm$ 5.31 days. There were no statistically significant differences in neonatal age, gestational age, and sex between the 2 groups.

The inclusion criteria were as follows: (I) the mothers of the newborns were21-34 years of age, healthy during pregnancy, and had no signs of infection; (II) the newborns had no history of intrauterine distress or postnatal asphyxia, gestational age was 37-42 weeks, and birth weight was 2,500-4,000 g; and (III) neonates with HBN met the diagnostic criteria of neonatal hyperbilirubinemia (12), that is, a total serum bilirubin (TSB) $>220.6 \mu \mathrm{mol} / \mathrm{L}$, transcutaneous bilirubin $(\mathrm{TCB})>12.9 \mathrm{mg} / \mathrm{dL}$ (in case of preterm infants, TSB $>256.5 \mu \mathrm{mol} / \mathrm{L}$ or $\mathrm{TCB}>15 \mathrm{mg} / \mathrm{dL}$ ), total daily increase in serum bilirubin $>85 \mu \mathrm{mol} / \mathrm{L}$, or serum conjugated bilirubin $>26 \mu \mathrm{mol} / \mathrm{L}$.

Exclusion criteria were as follows: (I) older than 28 days; (II) low-quality echocardiographic image, inability to obtain clear images, and not meeting the requirements of spot tracking and stratified strain data collection and analysis; (III) presence of congenital malformations; congenital immune deficiency; congenital heartdefects; severe infectious diseases, such as intrauterine virus infection, nervous system infection, sepsis, trisomy 21 , and other gene defects; and (IV) parents were not willing to participate in the study.

The study was conducted following the Declaration of Helsinki (as revised in 2013) and was approved by Ethics Committee of Affiliated Kunshan Hospital of Jiangsu University (No. 2017-03-021). Informed consent was obtained from the parents of all the neonates.

\section{Imaging methods}

\section{Conventional echocardiographic measurement}

Based on the measurement methods recommended by the American Society of Echocardiography (13), 2D, M-mode, and Doppler echocardiography were used to determine conventional left ventricular function parameters, including aortic sinus diameter (AOD), left atrial end-systolic diameter (LAD), left ventricular end-systolic diameter (LVED), LVED diameter, interventricular septal thickness, left ventricular posterior wall thickness, peak velocity of the pulmonary valve $\left(V_{\mathrm{PV}}\right)$, peak velocity of the aortic valve $\left(\mathrm{V}_{\mathrm{AV}}\right)$, peak velocity of the left ventricular outflow tract $\left(\mathrm{V}_{\mathrm{LVOT}}\right)$, peak velocity of the mitral valve $\left(V_{M V}\right)$, and peak velocity of the tricuspid valve $\left(V_{\mathrm{TV}}\right)$. The Simpson method was used to calculate LVEF and left fractional ventricular shortening (LVFS).

\section{DSTI layered strain technology}

Neonatal apical 4-chamber (4CH), left long ventricular axis (long apical axis, APLAX), and apical 2-chamber (2CH) 2D dynamic grayscale images were acquired according to the standard procedure. Three cardiac cycles were recorded separately and stored on the mobile hard drive in DICOM format. The stored 2D images were imported into the EchoPAC workstation and analyzed offline with the Qanalysis software using the 2D layered strain mode. First, the 2D grayscale image of the long axis view of the left ventricle was analyzed, and 3 points of the endocardium at the lateral mitral valve annulus, the apex, and the posterior mitral valve annulus were manually selected. The system automatically outlines the edge of the endocardium and determines the epicardium boundary, and the generated area of interest is synchronized with the ventricular wall. The system defaults to the ventricular septum, anterior ventricular septum, anterior wall, lateral wall, posterior wall, and lower wall. The inferior wall is equally divided into the basal segment, middle segment, and apical segment. For each segment, the subendocardial layer, mid-myocardial layer, and subepicardial layer, speckle tracking analysis was performed, and the longitudinal peak strain and LS curve of 

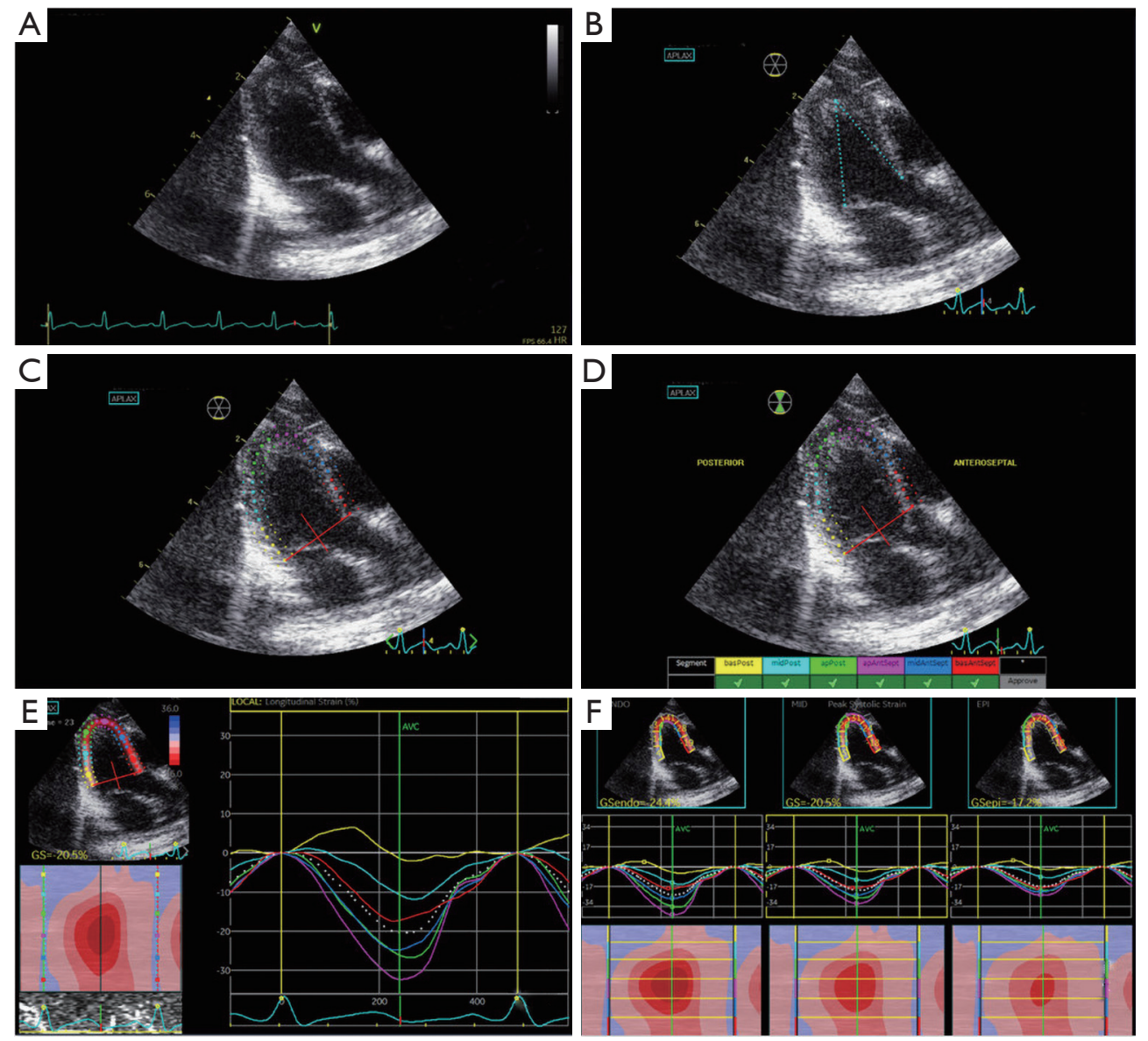

Figure 1 APLAX stratified strain measurement method. (A-C) Manually select the endocardium at the 3 points of the anterior septal mitral value annulus, the apex, and the posterior mitral valve annulus. The system automatically delineates the edge of the endocardium and determines the epicardial boundary. The generated area of interest moves synchronously with the ventricular wall. If the mosaic is poor, manually adjust to ensure that each segment can be tracked well. (D-F) By default, the system divides the anterior septum and posterior wall into the basal segment, middle segment, and apical segment. For each segment, the subintimal and mid-myocardium and subadventitial myocardium are spot-tracked and analyzed. Longitudinal peak strain and longitudinal strain curves of corresponding cardiac cycle are displayed.

the corresponding cardiac cycle were displayed (Figure 1). The apical $4 \mathrm{CH}$ view and the apical $2 \mathrm{CH}$ view were analyzed in the same way. All parameters were averaged over 3 cardiac cycles, the data were read and recorded, and the global longitudinal strain (GLS) of the 3layers of the myocardium in different ventricular walls, segments, and sections was calculated. All parameters were measured and analyzed by 2 attending physicians with extensive experience in ultrasound diagnosis, blinded to the specific grouping of the research participants. The dynamic images were stored in the EchoPAC workstation for measurement and analysis.

\section{Laboratory tests}

After admission, blood gas analysis, routine blood test, liver function, and myocardial enzyme spectrum were routinely performed in neonates with HBN. Test results were correlated with the ultrasound data.

\section{Statistical methods}

SPSS STATISTICS 23.0 (SPSS Inc., Chicago, IL, USA) was used for statistical processing. The measurement data conforming to the normal distribution, determined by 
Table 1 Comparison of clinical data and conventional echocardiographic parameters $(\bar{x} \pm \mathrm{s})$

\begin{tabular}{|c|c|c|c|c|}
\hline Parameters & Control group $(n=56)$ & Hyperbilirubinemia group $(n=134)$ & $\mathrm{t} / \chi^{2}$ value & $P$ value \\
\hline Apgar score & $9-10$ & $9-10$ & - & - \\
\hline Male/female & $25 / 31$ & $74 / 60$ & 1.620 & 0.203 \\
\hline Age (days) & $5.36 \pm 3.09$ & $6.25 \pm 3.79$ & 1.567 & 0.119 \\
\hline $\mathrm{BSA}\left(\mathrm{m}^{2}\right)$ & $0.22 \pm 0.01$ & $0.22 \pm 0.02$ & 1.263 & 0.208 \\
\hline TCB (mg/dL) & $8.82 \pm 2.42$ & $18.09 \pm 2.40^{\mathrm{a}}$ & 24.253 & $<0.001$ \\
\hline $\mathrm{AOD}(\mathrm{mm})$ & $8.23 \pm 0.83$ & $8.75 \pm 1.00^{\mathrm{a}}$ & 3.418 & 0.001 \\
\hline LAD (mm) & $11.09 \pm 1.37$ & $12.00 \pm 1.54^{\mathrm{a}}$ & 3.836 & $<0.001$ \\
\hline LVPWT (mm) & $3.30 \pm 1.16$ & $3.15 \pm 0.54$ & 1.252 & 0.212 \\
\hline LVEDs (mm) & $11.39 \pm 1.88$ & $12.00 \pm 2.47$ & 1.647 & 0.101 \\
\hline LVEF (\%) & $68.73 \pm 4.87$ & $68.58 \pm 5.57$ & 0.175 & 0.861 \\
\hline LVFS (\%) & $36.44 \pm 4.87$ & $35.97 \pm 4.59$ & 0.675 & 0.500 \\
\hline $\mathrm{V}_{\mathrm{PV}}(\mathrm{m} / \mathrm{s})$ & $0.94 \pm 0.11$ & $0.96 \pm 0.11$ & 0.582 & 0.561 \\
\hline $\mathrm{V}_{\mathrm{AV}}(\mathrm{m} / \mathrm{s})$ & $0.86 \pm 0.14$ & $0.93 \pm 0.13^{\mathrm{a}}$ & 2.974 & 0.003 \\
\hline $\mathrm{V}_{\text {LVOT }}(\mathrm{m} / \mathrm{s})$ & $0.52 \pm 0.06$ & $0.59 \pm 0.09^{a}$ & 5.253 & $<0.001$ \\
\hline $\mathrm{V}_{\mathrm{MV}}(\mathrm{m} / \mathrm{s})$ & $0.57 \pm 0.04$ & $0.64 \pm 0.08^{\mathrm{a}}$ & 5.800 & $<0.001$ \\
\hline
\end{tabular}

Compared with the control group, ${ }^{a} \mathrm{P}<0.05$. AOD, aortic sinus diameter; IVSD, interventricular septal thickness diameter; LAD, left atrial end-systolic diameter; LVED, left ventricular end-systolic diameter; LVEDd, left ventricular end-diastolic diameter; LVEF, left ventricular ejection fraction; LVFS, left ventricular fractional shortening rate; LVPWT, left ventricular posterior wall thickness; TCB, transcutaneous bilirubin; $\mathrm{V}_{\mathrm{AV}}$, peak velocity of the aortic valve; $\mathrm{V}_{\mathrm{LVOT}}$, peak velocity of the left ventricular outflow tract; $\mathrm{V}_{\mathrm{MV}}$, peak velocity of the mitral valve; $\mathrm{V}_{\mathrm{PV}}$, peak velocity of the pulmonary valve; $\mathrm{V}_{\mathrm{TV}}$, peak velocity of the tricuspid valve.

the single-sample Kolmogorov-Smirnov normality test, are expressed as the mean \pm standard deviation $(\bar{x} \pm \mathrm{s})$. The 2 groups were compared using independent samples $t$-test, while comparisons among 3 or more groups were made using a 1-way analysis of variance. The LSD (Least-Significant Difference) method was used for pairwise comparison if the difference was statistically significant. For data not conforming to the normal distribution, the median \pm interquartile range was used. The Mann-Whitney U-test was employed to compare 2 groups, and the Kruskal-Wallis H-test was used when comparing 3 groups. Enumeration data are expressed as fractions, and comparisons between groups were made using $\chi^{2}$-test. Pearson correlation analysis was used for continuous data. $\mathrm{P}<0.05$ indicated statistical significance.

\section{Results}

\section{Basic clinical data and conventional echocardiographic parameters}

Compared with the control group, the values of TCB, AOD, LAD, VAV, VLVOT, VMV, and VTV were significantly higher in the HBN group $(\mathrm{P}<0.05)$ than in the control group. There were no statistically significant differences in LVEF, LVFS, gestational age, sex ratio, age, weight, and Apgar score at birth between the 2 groups $(\mathrm{P}>0.05)$ (Table 1). 
Table 2 Comparison of different stratified strains of the left ventricular wall between groups $(\bar{x} \pm s)$

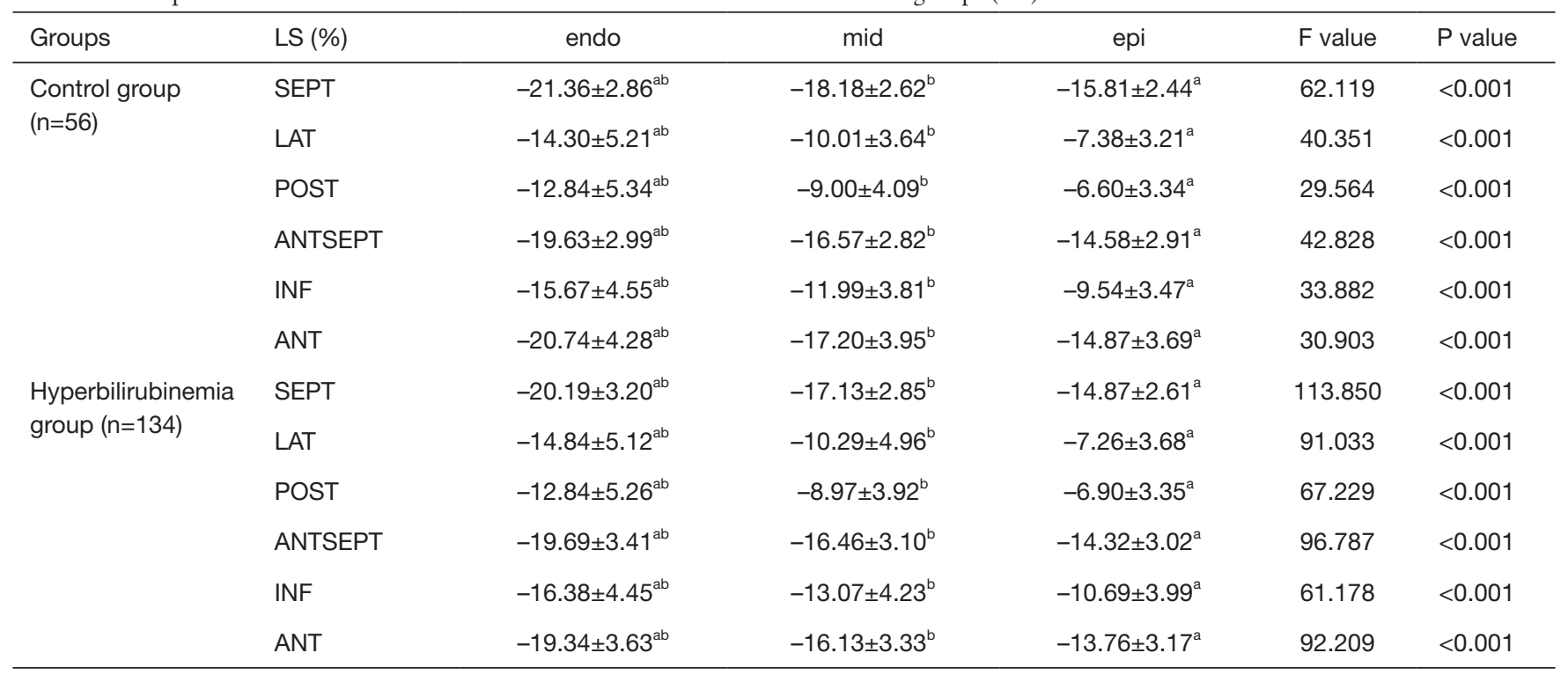

Compared with mid, ${ }^{\mathrm{a}} \mathrm{P}<0.05$; compared with epi, ${ }^{\mathrm{b}} \mathrm{P}<0.05$. ANT, anterior wall; ANTSEPT, anterior partition; endo, subintimal; epi, subadventitial; INF, inferior wall; LAT, side wall; LS, longitudinal strain; mid, middle; POST, posterior wall; SEPT, interventricular septum.

Table 3 Comparison of stratified strains in different segments of the left ventricle $(\bar{x} \pm s)$

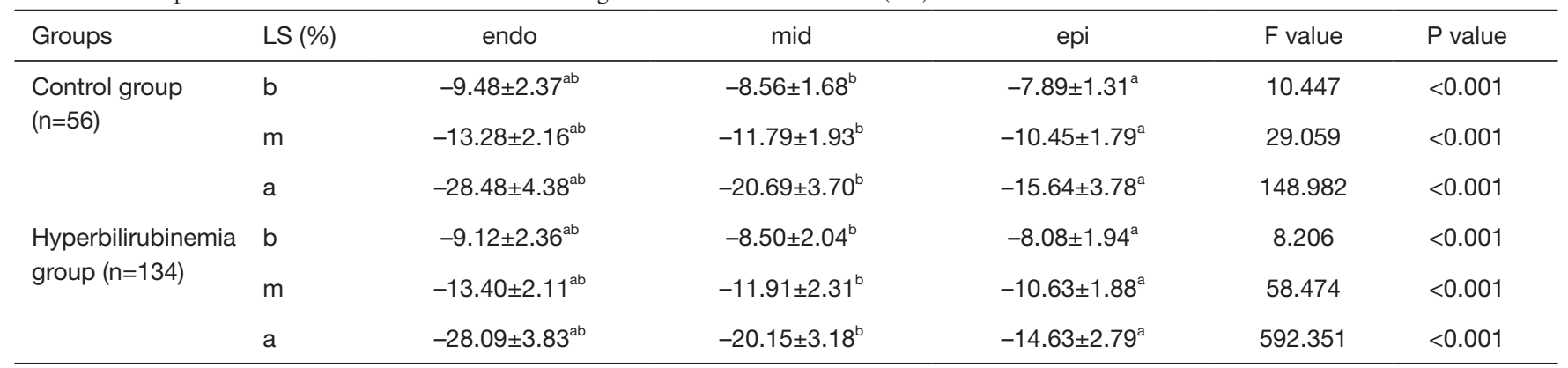

Compared with mid, ${ }^{a} \mathrm{P}<0.05$; compared with epi, ${ }^{\mathrm{b}} \mathrm{P}<0.05$. a, apical segment; b, basal segment; endo, subintimal; epi, subadventitial; LS, longitudinal strain; $\mathrm{m}$, middle segment; mid, middle.

\section{Longitudinal stratified strain parameters}

A total of 10,260 ( 190 cases $\times 18$ segments $\times 3$ layers) stratified myocardium segments were tracked in all neonates in the present study. Of these, 397 segments that were not recognized due to unclear $2 \mathrm{D}$ images and poor tracking and display by the software were excluded. Therefore, the tracking success rate was $96.13 \%$.

\section{Stratified strain in different segments of the left ventricular wall}

The LS of the ventricular septum, sidewall, posterior wall, anterior septum, inferior wall, and anterior wall decreased sequentially from the subendocardial to the middle and to the subadventitial myocardium in both groups. All changes were statistically significant $(\mathrm{P}<0.05)$ (Table 2).

\section{Stratified strain in different segments of the left ventricle}

The LS of the basal, middle, and apical segments decreased sequentially from the subendocardial to the middle and to the subadventitial myocardium in both groups, and the differences were statistically significant $(\mathrm{P}<0.05)$ (Table 3).

The overall stratified strain of the left ventricle

The GLS of the left ventricle decreased from the 
Table 4 Intragroup comparison of overall stratified strainsin the left ventricle $(\bar{x} \pm \mathrm{s})$

\begin{tabular}{|c|c|c|c|c|c|}
\hline Groups & GLSendo (\%) & GLSmid (\%) & GLSepi (\%) & F value & $P$ value \\
\hline Hyperbilirubinemia group $(n=134)$ & $-17.35 \pm 2.44^{\mathrm{ab}}$ & $-13.67 \pm 2.07^{b}$ & $-11.30 \pm 1.87^{\mathrm{a}}$ & 270.955 & $<0.001$ \\
\hline
\end{tabular}

Compared with GLSmid, ${ }^{\mathrm{a}} \mathrm{P}<0.05$; compared with GLSepi, ${ }^{\mathrm{b}} \mathrm{P}<0.05$. endo, subintimal; epi, subadventitial; GLS, global longitudinal strain; mid, middle.

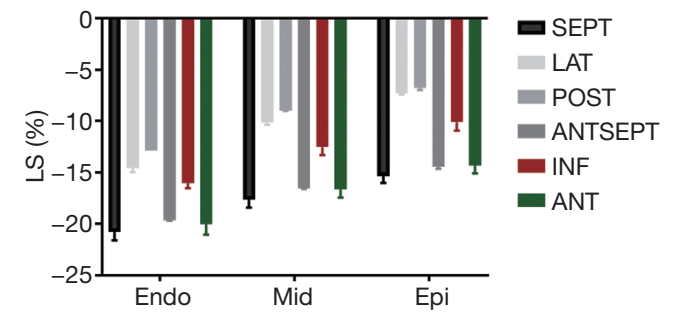

Figure 2 Comparison of different wall strain groups in each layer of the left ventricle. ANT, anterior wall; ANTSEPT, anterior partition; endo, subintimal; epi, subadventitial; INF, inferior wall; LAT, side wall; LS, longitudinal strain; mid, middle; POST, posterior wall; SEPT, interventricular septum.

subendocardial to the middle to the subpericardial myocardium in both groups, and the differences were statistically significant $(\mathrm{P}<0.05)$ (Table 4).

\section{Wall strains in each layer of the left ventricle}

The difference in stratified LS between the 2 groups was statistically significant $(\mathrm{P}<0.05)$ in most wall segments. The 2 groups of wall LS of the ventricular septum, anterior wall (or anterior septum), inferior wall, lateral wall, and posterior wall, showed a decreasing trend (Figure 2).

\section{Strains in different segments of each layer of the left ventricle}

The stratified LS gradually increased from the basal to the apical segment in both groups, and the differences were statistically significant $(\mathrm{P}<0.05)$ (Figure 3).

\section{Longitudinal stratified strain parameters in the control and $\mathrm{HBN}$ groups}

Comparison of stratified strains of different segments of the left ventricular wall

Compared with the control group, the LS of the ventricular septal myocardium was significantly reduced in the HBN group $(\mathrm{P}<0.05)$. The LS of the subintimal and mid-

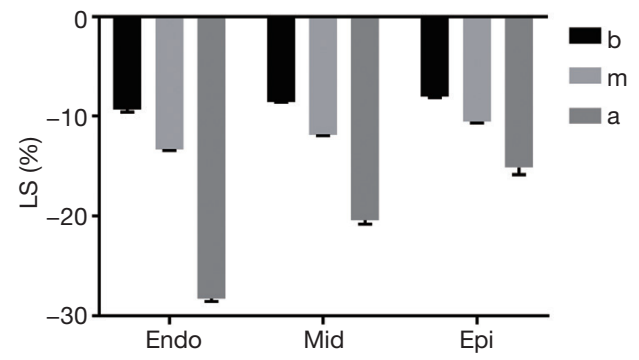

Figure 3 Comparison of different segments in each layer of the left ventricle. a, apical segment; b, basal segment; endo, subintimal; epi, subadventitial; LS, longitudinal strain; mid, middle; $\mathrm{m}$, the middle segment.

myocardium of the lateral wall increased, while the LS of the subadventitial myocardium decreased, but these differences were not statistically significant $(\mathrm{P}>0.05)$. The LS of the posterior mid-myocardium decreased, and the LS of the subadventitial myocardium increased, but the difference was not statistically significant $(P>0.05)$. The LS of the subintimal anterior septum increased but decreased in the mid-myocardium and subadventitial myocardium; however, these differences were not statistically significant $(\mathrm{P}>0.05)$. The LS of the 3 layers of the inferior myocardium increased, but the difference was not statistically significant $(\mathrm{P}>0.05)$. The LS in the layers of the anterior myocardium decreased, and a statistically significant difference was found between the subintima and subpericardium $(\mathrm{P}<0.05)$ (Figure 4).

\section{Stratified strain in different segments of the left ventricle}

In comparison with the control group, the LS of the basal segment of the endocardium and the mid-myocardium decreased in the HBN group, and LS of the subadventitial myocardium increased; however, these differences were not statistically significant $(\mathrm{P}>0.05)$. The LS of the 3 layers of the apical segment of the myocardium was reduced, but only the difference in the LS of the subadventitial myocardium was statistically significant $(\mathrm{P}<0.05)$ (Figure 5). 


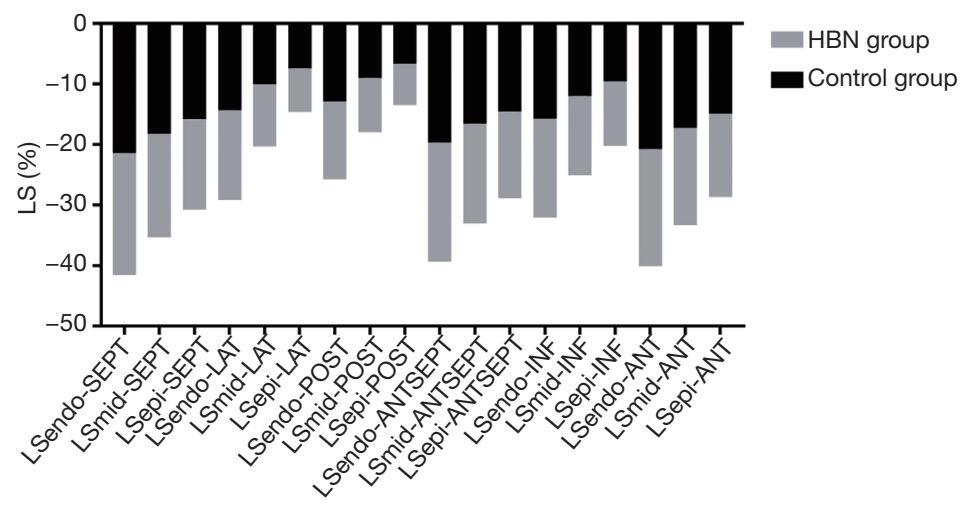

Figure 4 Comparison of different stratified strains in the left ventricle. ANT, anterior wall; ANTSEPT, anterior partition; endo, subintimal; epi, subadventitial; HBN, hyperbilirubinemia; INF, inferior wall; LAT, side wall; LS, longitudinal strain; mid, middle; POST, posterior wall; SEPT, interventricular septum.

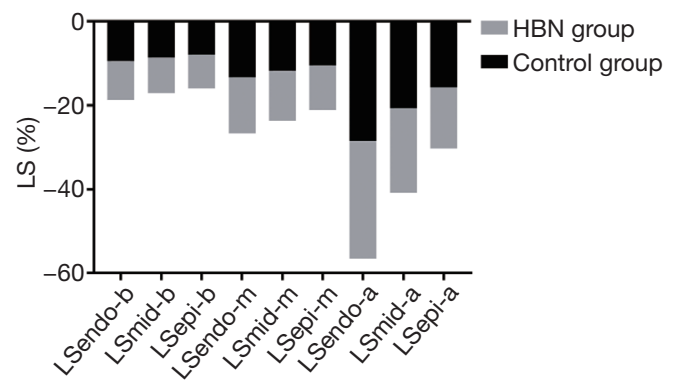

Figure 5 Comparison of the stratified strain in different segments of the left ventricle between the 2 groups. a, apical segment; b, basal segment; endo, subintimal; epi, subadventitial; HBN, hyperbilirubinemia; m, middle segment; mid, middle; LS, longitudinal strain.

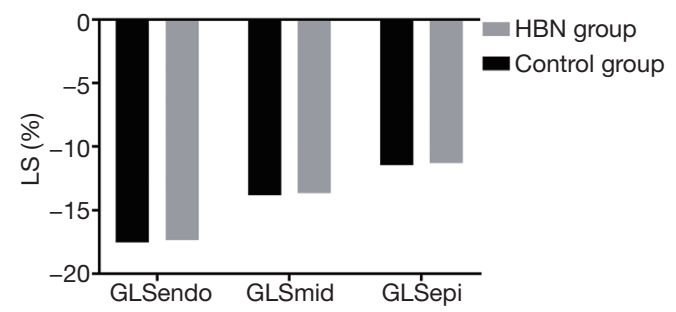

Figure 6 Comparison of the global longitudinal stratified strain between the control group and the HBN group. GLS, global longitudinal strain; endo, subintimal; epi, subadventitial; HBN, hyperbilirubinemia; mid, middle.

The overall stratified strain of the left ventricle

In comparison with the control group, the GLS of the subintimal, mid-myocardium, and subadventitial myocardium was reduced in the HBN group, but the difference was not statistically significant $(\mathrm{P}>0.05)$ (Figure 6).

\section{Correlation analysis}

Pearson correlation analysis showed that serum $\mathrm{pH}$ was positively correlated with the LS of the posterior wall of the left ventricle, the left ventricular media, and adventitia of the left ventricular 3 -chamber $(3 \mathrm{CH})$ view, and the intima and adventitia of the middle segment $(\mathrm{r}=0.28-0.39$, all $\mathrm{P}<0.05$ ). The percentage of reticulocytes ( $\mathrm{RC}$ ) was negatively correlated with the LS of the posterior intima, anterior intima, 3-lumen intima, anterior media, and adventitia basal segment $(\mathrm{r}=-0.24$ to -0.28 , all $\mathrm{P}<0.05)$. Aspartate aminotransferase (AST) was negatively correlated with the LS of the intimal septum, media septum, adventitial septum, and adventitial basal segment $(\mathrm{r}=-0.18$ to -0.26 , all $\mathrm{P}<0.05)$. Alanine aminotransferase (ALT) was negatively correlated with LS in the lateral wall of the intima and the lateral wall of the media $(r=-0.22$ to -0.27 , both $\mathrm{P}<0.05)$. Lactate dehydrogenase $(\mathrm{LDH})$ was negatively correlated with LS of the adventitial septum, basal media, and adventitial basal segment $(\mathrm{r}=-0.18$ to -0.24 , all $\mathrm{P}<0.05)$. Total bilirubin (TBIL) was negatively correlated with the LS of the adventitia of the ventricular septum and the adventitia on the $4 \mathrm{CH}$ view of the heart. Indirect bilirubin (IBIL) was negatively correlated with the LS of the adventitia, $4 \mathrm{CH}$ adventitia, and $3 \mathrm{CH}$ media $(\mathrm{r}=-0.17$ to -0.24 , all $\mathrm{P}<0.05$ ). Creatine kinase (CK) was negatively correlated with the LS of the posterior media wall, posterior wall of adventitia, and $3 \mathrm{CH}$ adventitia $(\mathrm{r}=-0.18$ to -0.22 , all $\mathrm{P}<0.05)$. There was 
Table 5 Correlation analysis of layered longitudinal strain in hyperbilirubinemia group and laboratory examination results

\begin{tabular}{|c|c|c|c|}
\hline Index & Strain parameters & $r$ value & $P$ value \\
\hline \multirow[t]{7}{*}{$\mathrm{pH}$} & LSendo-POST & 0.280 & 0.039 \\
\hline & LSmid-POST & 0.379 & 0.004 \\
\hline & LSmid-APLAX & 0.299 & 0.026 \\
\hline & LSepi-POST & 0.397 & 0.003 \\
\hline & LSepi-APLAX & 0.309 & 0.022 \\
\hline & LSmid-m & 0.299 & 0.027 \\
\hline & LSepi-m & 0.308 & 0.022 \\
\hline \multirow[t]{5}{*}{$\mathrm{RC}$} & LSendo-POST & -0.228 & 0.048 \\
\hline & LSendo-ANTSEPT & -0.254 & 0.027 \\
\hline & LSendo-APLAX & -0.279 & 0.015 \\
\hline & LSmid-ANTSEPT & -0.231 & 0.045 \\
\hline & LSepi-b & -0.241 & 0.036 \\
\hline \multirow[t]{4}{*}{ AST } & LSendo-SEPT & -0.241 & 0.029 \\
\hline & LSmid-SEPT & -0.219 & 0.048 \\
\hline & LSepi-SEPT & -0.264 & 0.016 \\
\hline & LSepi-b & -0.179 & 0.036 \\
\hline \multirow[t]{2}{*}{ ALT } & LSendo-LAT & -0.270 & 0.014 \\
\hline & LSmid-LAT & -0.219 & 0.048 \\
\hline \multirow[t]{3}{*}{$\mathrm{LDH}$} & LSepi-SEPT & -0.242 & 0.028 \\
\hline & LSmid-b & -0.180 & 0.037 \\
\hline & LSepi-b & -0.221 & 0.010 \\
\hline \multirow[t]{2}{*}{ TBIL } & LSepi-SEPT & -0.223 & 0.045 \\
\hline & LSepi-4CH & -0.239 & 0.032 \\
\hline \multirow[t]{3}{*}{ IBIL } & LSepi-SEPT & -0.232 & 0.037 \\
\hline & LSepi-4CH & -0.242 & 0.029 \\
\hline & LSmid-APLAX & -0.170 & 0.049 \\
\hline \multirow[t]{3}{*}{ CK } & LSmid-POST & -0.178 & 0.046 \\
\hline & LSepi-POST & -0.215 & 0.015 \\
\hline & LSepi-APLAX & -0.204 & 0.021 \\
\hline
\end{tabular}

ALT, alanine aminotransferase; ANT, anterior wall; ANTSEPT, anterior partition; APLAX, apical long-axis view; AST, aspartate aminotransferase; b, basal segment; CK, creatine kinase; endo, subintimal; epi, subadventitial; IBIL, indirect bilirubin; INF, inferior wall; $\mathrm{LDH}$, lactate dehydrogenase; $\mathrm{m}$, middle segment; mid, middle; POST, posterior wall; LAT, side wall; LS, longitudinal strain; RC, reticulocytes; SEPT, interventricular septum; $2 \mathrm{CH}$, apical 2-chamber view; $4 \mathrm{CH}$, 4-chamber view. no significant correlation between CK isoenzyme and LS of each layer of the left ventricle (all $\mathrm{P}>0.05$ ) (Table 5).

\section{Discussion}

Bilirubin is the most important product of hemoglobin decomposition in the human body, and approximately $80 \%$ of bilirubin originates from the hemoglobin of aging red blood cells (14). The metabolism of bilirubin in the neonatal period differs from that in adults (15). More than half of newborns experience varying degrees of the increase in serum bilirubin, which can cause yellowing of the skin, mucus membranes, sclera, and other tissues. This condition is called neonatal jaundice. Animal experimental studies have demonstrated that a mild increase in bilirubin could improve myocardial stress resistance, reduce the risk of cardiovascular events and, at the same time, increase the activity of superoxide dismutase (SOD) in myocardial cells and reduce the content of malondialdehyde. Bilirubin can scavenge oxygen free radicals in cardiomyocytes, protects from oxidative damage, and has a variety of other beneficial physiological functions promoting the metabolism of newborns (16). However, when bilirubin increases to concentrations $>220.6 \mu \mathrm{mol} / \mathrm{L}(12.9 \mathrm{mg} / \mathrm{dL})$, or $256.5 \mu \mathrm{mol} / \mathrm{L}(15 \mathrm{mg} / \mathrm{dL})$ in premature infants, HBN ensues. It is widely believed that neonatal cardiomyocytes have a lower reserve capacity than adult cells and less effective compensatory regulation (17). Therefore, HBN is one of the common causes of myocardial injury in neonates.

2DSTI is based on 2D grayscale images. It tracks and records the motion trajectory of the speckle echo during the contraction and relaxation of the myocardial tissue in the region of interest, frame by frame, and calculates the displacement amplitude of the speckle and myocardium in the entire region of interest. The displacement amplitude of the spots and the degree of myocardial deformation during segmental myocardial movement can sensitively reflect small changes in myocardial contraction movement (18). This technology has a good spatial and temporal resolution and overcomes the angular dependence of the tissue Doppler technology, and is relatively independent of the front and rear load. 2DSTI can sensitively and accurately identify the myocardial segment with abnormal motion, which can be used for the early detection of pathological changes. 2DSTI is an important tool for determining the impairment of myocardial function and has a good correlation with the results of cardiac performance measured by magnetic 
resonance imaging and digital subtraction angiography $(19,20)$. In recent years, this technology has been used to evaluate left ventricular function in neonates with heart disease, and its sensitivity is higher than that of conventional echocardiography $(21,22)$.

The myocardial band theory proposes that each layer of the myocardium has different anatomical characteristics. In addition to the distinct roles of each layer of the myocardium in the left ventricular diastolic and systolic movements, certain diseases in their early or acute phase may impact the strain of each layer of the left ventricle differently, causing loss of structural integrity of the layered sand local myocardial damage. Stratified strain technology accurately evaluates myocardial deformation and function changes at different levels and directly identifies the location and degree of occult myocardial injury. This technology is based on the development of 2DSTI that simultaneously and continuously tracks the stable acoustic spots in the subintimal, mid-, and subadventitial myocardium to quantitate the contractile strain and the overall strain of the heart in 18 segments of the left ventricle in 3 longaxis views. This information is then used to evaluate the deformation and functional changes of each layer of the myocardium (23). Compared with the traditional overall assessment of the ventricular wall, stratified strain technology can more accurately identify the 3-layer anatomy of the left ventricular myocardium, providing adequate means for the early detection of subclinical myocardial injury. This technology has broader clinical application prospects and numerous advantages. It has been used to evaluate the myocardial mechanical state in patients with coronary heart disease, hypertension, myocardial amyloidosis, and other diseases and in healthy adults (24).

\section{Comparison with conventional echocardiographic test results}

The present study results indicate that the inner diameters of the aortic sinus and left atrium and the flow velocities of the mitral, tricuspid, and aortic valves and left ventricular outflow tract in the HBN group were higher than those in the control group. Although the values were within the normal range, the difference was statistically significant $(\mathrm{P}<0.05)$. These findings indicate that the body of HBN neonates is in a higher metabolic state and stress state, the left ventricular volume load is significantly elevated, and the flow velocity of each valve orifice is increased. LVEF, reflecting the global left ventricular systolic function, becomes abnormal only when cardiomyocytes are severely damaged or the disease is relatively advanced (25). Myocardial injury is difficult to reverse at that time. The LVEF in the HBN group was in the normal range and was not statistically different from that of the control group, indicating that the overall left ventricular systolic function was not affected by HBN. Therefore, the LVEF measurement cannot detect the difference in left ventricular systolic function between the 2 groups.

\section{Comparison of myocardial longitudinal stratified strain detection results}

\section{Characteristics of the stratified strain of left ventricular myocardium in newborns}

The results indicated that, regardless of the presence of hyperbilirubinemia in the neonates, the strain curves of the 18 segments of the left ventricular wall in the longitudinal direction were approximately the same, that is, myocardial shortening during the systole is represented by a negative trend curve below the 0 lines, and a positive trend curve represents myocardial elongation during the diastole. The strain of each ventricular wall and wall segment, and the overall stratified strain, all have the same gradient characteristics, which are essentially the same as in normal adults and neonates; that is, myocardial LS decreases from the subendocardial to subepicardial myocardium $(\mathrm{P}<0.05)$. This transmural difference in the strain may be related to the different radii of curvature in each myocardial layer and different local myocardial strain tensions. Anatomically, the normal left ventricular wall myocardium is not uniform, and the 3 layers of myocardial fibers are dissected in a spiral pattern. The 3 layers of the myocardium with different structures comprising the left ventricle are folded onto each other and correspond to the endocardial myocardium with the right-handed spiral arrangement, annular middle myocardium perpendicular to the long axis of the heart, and epicardial myocardium with a left-hand spiral arrangement. The differences in strain between the segments are statistically significant (26-28). The LS increases from the basal segment to the apex of the heart, as also indicated by Addetia et al.'s study (29). A possible reason for this increase is that each segment of the left ventricle faces the geometric center near the apex during systole and moves away from the center during diastole (30). Therefore, the basal segment has the smallest deformation, and the apical segment has the largest. At the same time, the myocardial fibers at the base of the left ventricle travel in a circular direction. This 
pattern is dominant, and since the myocardial fibers in the apex are dominant in oblique movement, the LS value in the apical segment will be slightly higher than in other segments, which may be related to the apparent advantage of the oblique fiber distribution. The LS of the ventricular septum, anterior wall (or anterior septum), inferior wall, sidewall, and posterior wall of the myocardium showed a decreasing trend $(\mathrm{P}<0.05)$, a result similar to those obtained in previous studies (31). The uneven deformation and the gradient change of LS in each ventricular wall, segment, and a layer of the myocardium, as well as the maintenance of the longitudinal strain gradient, are essential for coordinating the overall left ventricular contraction movement and the maintenance of the normal ejection function (32).

\section{Vertical stratified strain in neonates with $\mathrm{HBN}$}

The present study's findings indicated that there were segmental differences in the effect of HBN on myocardial contractility in neonates. The LS of the full thickness of the ventricular septum and the inferior intima and adventitia of the anterior wall myocardium was significantly decreased in HBN $(\mathrm{P}<0.05)$. The reason for this change may be related to coronary hemodynamics and cardiac anatomy. The left anterior descending coronary artery supplies the anterior wall. The left anterior descending branch and its branches provide more than $50 \%$ of the blood supply of the left ventricular wall. Pinto and Campos showed that the blood flow along the wall of the left anterior descending branch vessel wall gradually decreases as the blood vessel extends to the distal end, and lower shear stress can inhibit the function of vascular endothelial cells, making them more susceptible to chemical stimulation (33). Therefore, in the presence of HBN, the left anterior descending branch vessel is more susceptible to injury than other coronary arteries. If the disease-related changes are severe, blood flow stasis often occurs in areas with lower wall shear stress, and toxic substances are more likely to linger for a longer time. This condition aggravates a local myocardial injury, resulting in a localized decrease in LS. In addition, the ventricular septum and the anterior wall are closer to the right ventricle than other ventricular walls, and the right ventricular wall is thinner and more compliant. The ventricular septum and anterior wall have low tolerance and compensatory power for load changes and myocardial toxicity (34). The comparison among segments showed that the LS of the 3-layer myocardium in the apical segment was lower in the HBN group than in the control group, and the difference in the adventitial apical segment was statistically significant $(\mathrm{P}<0.05)$. Considering that the apical segment has a lower blood supply than the basal segment of the left ventricle, its contractility may be the first to be damaged by cardiotoxicity.

Previously published studies have indicated that the subendocardial myocardium bears greater wall tension and work than the mid-myocardium and subadventitial myocardium and is more susceptible to adverse factors, such as ischemia and hypoxia toxicity, and metabolism $(24,35)$. At the early stage of the impairment of systolic function, the damage mostly affects the endomyocardium. However, the findings in the present study indicated that the wall and segmental LS were significantly reduced in the HBN group. Myocardial damage was concentrated in the intimal and adventitial regions, while the mid-myocardium was less affected. The likely reason for this difference is that longitudinal myocardial fibers are mainly present in the subendocardial and subepicardial myocardium of the left ventricular wall. These longitudinal myocardial fibers innervate the corresponding myocardium showing longitudinal motion, while the middle layer is composed of circular fibers responsible for the circular motion of the myocardium. Therefore, the LS sensitivity of the subendocardial and subpericardial regions is higher than that of the middle myocardium (36).

The GLS represents the average strain value of each ventricular wall segment along longitudinal fibers and represents an index of the overall left ventricular systolic function. Although the GLS of each layer in the HBN group was lower than that in the control group, this difference was not statistically significant $(P>0.05)$, as the 3 layers of the heart muscle fibers pull each other and cooperate. The deformation of different muscle layers is a combined effect of their actions and the deformation of the adjacent myocardium (37). In the current study, neonates in the HBN group showed a decrease in the strain in only part of the ventricular wall, reflecting segmental myocardium damage, while other parts of the myocardium were subjected to an increased stratified strain. The segmental character of the injury limits the damage to the ventricular wall, which a compensatory enhancement of contraction can counteract. This phenomenon also explains why the local contractile function of the myocardium in neonates in the HBN group was impaired while the LVEF remained within the normal range. It shows that myocardial damage in neonates with HBN is relatively mild and does not result in any obvious clinical manifestations, such as arrhythmia or 
cardiac insufficiency, further demonstrating that myocardial longitudinal stratified strain is a more sensitive index of myocardial function compared with LVEF.

\section{Correlation of stratified strain with laboratory indicators}

Several longitudinal stratified strain parameters of the left ventricle correlated with serum $\mathrm{pH}$, the percentage of RC, AST, ALT, LDH, muscle CK, TBIL, and IBIL, but the correlation was not statistically significant (all $\mathrm{r}<0.5)$. Previously published studies demonstrated that bilirubin at high concentrations could change cell membrane permeability, enter the cytoplasm and nucleus, and interfere with the normal oxidation reaction in the mitochondria, affecting the energy metabolism of the cell $(33,34)$. Furthermore, bilirubin can reduce SOD activity, resulting in a significant accumulation of oxidative free radicals. Bilirubin increases the red blood cell aggregation index, decreases the red blood cell deformation index, and increases plasma viscosity, slowing down blood flow. The latter effect triggers microcirculation disorders and aggravates tissue hypoxia.

Additionally, at excessive concentrations, bilirubin binds plasma albumin and affects metabolism by antagonizing molecules that need to be combined with albumin for their activity. Importantly, HBN is induced by various factors, such as hemolysis, infection, and breastfeeding, which can cause complex and diverse pathological changes, such as acidosis and liver damage (35). Therefore, myocardial contractility is affected by many factors capable of increasing the concentration of bilirubin. Moreover, increased bilirubin may not be the only cause of myocardial damage, and the mechanisms underlying myocardial injury remain to be further explored.

\section{Limitations}

The present study has some limitations. First, 2DSTI layered strain technology has high requirements for image clarity and relies on a higher frame rate. Because the intercostal space in newborns is narrower and more difficult to access than in adults, obtaining clear images on the short axis was problematic, and the circumferential strain could not be assessed. Second, the system software automatically performs linear average layered imaging analysis of the myocardium, which may not correspond precisely to the cardiac tissue's actual anatomical layers. Myocardial movements occur in $3 \mathrm{D}$, but the study utilized 2D layered strain imaging to track the planar myocardial spots, limiting to a certain extent the accuracy of the measurements of myocardial movement. Third, follow up of neonates with HBN was not performed, and the long-term outcomes of the effect of HBN on the contractile function of the myocardium require further research. In the future, these are still the main research directions of our research team, and we will continue to work hard for this.

\section{Acknowledgments}

Funding: None.

\section{Footnote}

Conflicts of Interest: All authors have completed the ICMJE uniform disclosure form (available at https://dx.doi. org/10.21037/qims-21-197). The authors have no conflicts of interest to declare.

Ethical Statement: The authors are accountable for all aspects of the work in ensuring that questions related to the accuracy or integrity of any part of the work are appropriately investigated and resolved. The study was conducted following the Declaration of Helsinki (as revised in 2013) and was approved by Ethics Committee of Affiliated Kunshan Hospital of Jiangsu University (No. 2017-03-021).Informed consent was obtained from the parents of all the neonates.

Open Access Statement: This is an Open Access article distributed in accordance with the Creative Commons Attribution-NonCommercial-NoDerivs 4.0 International License (CC BY-NC-ND 4.0), which permits the noncommercial replication and distribution of the article with the strict proviso that no changes or edits are made and the original work is properly cited (including links to both the formal publication through the relevant DOI and the license). See: https://creativecommons.org/licenses/by-nc-nd/4.0/.

\section{References}

1. Farouk ZL, Usman F, Musa BM, Ezeaka VC, Okolo A. Societal awareness on neonatal hyperbilirubinemia: A systematic review and meta-analysis. Semin Perinatol 2021;45:151361.

2. Alkén J, Håkansson S, Ekéus C, Gustafson P, Norman M. Rates of Extreme Neonatal Hyperbilirubinemia and 
Kernicterus in Children and Adherence to National

Guidelines for Screening, Diagnosis, and Treatment in Sweden. JAMA Netw Open 2019;2:e190858.

3. Zhou Z, Li X, Chen X, Yao L, Pan C, Huang H, Luo F, Zheng X, Sun X, Tan F. Comparison of P1 and 16S rRNA genes for detection of Mycoplasma pneumoniae by nested PCR in adults in Zhejiang, China. J Infect Dev Ctries 2015;9:244-53.

4. Yu H, Zou L, He Y, Luo L, Dong W, Zhang Y, Lei $\mathrm{X}$. Associations between neonatal serum bilirubin and childhood hypertension. PLoS One 2019;14:e0219942.

5. Philouze C, Obert P, Nottin S, Benamor A, Barthez O, Aboukhoudir F. Dobutamine Stress Echocardiography Unmasks Early Left Ventricular Dysfunction in Asymptomatic Patients with Uncomplicated Type 2 Diabetes: A Comprehensive Two-Dimensional SpeckleTracking Imaging Study. J Am Soc Echocardiogr 2018;31:587-97.

6. Torrent-Guasp F, Kocica MJ, Corno A, Komeda M, Cox J, Flotats A, Ballester-Rodes M, Carreras-Costa F. Systolic ventricular filling. Eur J Cardiothorac Surg 2004;25:376-86.

7. Torrent-Guasp F, Ballester M, Buckberg GD, Carreras F, Flotats A, Carrió I, Ferreira A, Samuels LE, Narula J. Spatial orientation of the ventricular muscle band: physiologic contribution and surgical implications. J Thorac Cardiovasc Surg 2001;122:389-92.

8. Klaeboe LG, Haland TF, Leren IS, Ter Bekke RMA, Brekke PH, Røsjø H, Omland T, Gullestad L, Aakhus S, Haugaa KH, Edvardsen T. Prognostic Value of Left Ventricular Deformation Parameters in Patients with Severe Aortic Stenosis: A Pilot Study of the Usefulness of Strain Echocardiography. J Am Soc Echocardiogr 2017;30:727-735.e1.

9. Hwang IC, Cho GY, Yoon YE, Park JJ. Association Between Global Longitudinal Strain and Cardiovascular Events in Patients With Left Bundle Branch Block Assessed Using Two-Dimensional Speckle-Tracking Echocardiography. J Am Soc Echocardiogr 2018;31:52-63.e6.

10. Kim SA, Kim MN, Shim WJ, Park SM. Layer-specific dyssynchrony and its relationship to the change of left ventricular function in hypertensive patients. Heart Vessels 2016;31:528-34.

11. Shi J, Xing Y, Qian J, Yang H, Yan Y, Li Q, Luo L, Kong D, Pan C, Shu X. Early Assessment of Left Ventricular Function by Layer-Specific Strain and Its Relationship to Pulsatile Arterial Load in Patients with Coronary Slow Flow. Int Heart J 2019;60:586-92.

12. Morioka I. Hyperbilirubinemia in preterm infants in
Japan: New treatment criteria. Pediatr Int 2018;60:684-90.

13. Recommendations for Cardiac Chamber Quantification by Echocardiography in Adults: An Update from the American Society of Echocardiography and the European Association of, Cardiovascular Imaging. Eur Heart J Cardiovasc Imaging 2016;17:412.

14. Jasprova J, Dal Ben M, Vianello E, Goncharova I, Urbanova M, Vyroubalova K, Gazzin S, Tiribelli C, Sticha M, Cerna M, Vitek L. The Biological Effects of Bilirubin Photoisomers. PLoS One 2016;11:e0148126.

15. Olusanya BO, Kaplan M, Hansen TWR. Neonatal hyperbilirubinaemia: a global perspective. Lancet Child Adolesc Health 2018;2:610-20.

16. Fujiwara R, Haag M, Schaeffeler E, Nies AT, Zanger UM, Schwab M. Systemic regulation of bilirubin homeostasis: Potential benefits of hyperbilirubinemia. Hepatology 2018;67:1609-19.

17. Sansbury BE, Jones SP, Riggs DW, Darley-Usmar VM, Hill BG. Bioenergetic function in cardiovascular cells: the importance of the reserve capacity and its biological regulation. Chem Biol Interact 2011;191:288-95.

18. Auriau J, Truong BL, Douchin S, Bouvaist H, Michalowicz G, Usson Y, Jouk PS. Longitudinal Study by TwoDimensional Speckle-Tracking Echocardiography of the Left Ventricle Rotational Mechanics during Postnatal Adaptation in Healthy Newborns. J Am Soc Echocardiogr 2020;33:252-4.

19. Caspar T, Fichot M, Ohana M, El Ghannudi S, Morel O, Ohlmann P. Late Detection of Left Ventricular Dysfunction Using Two-Dimensional and ThreeDimensional Speckle-Tracking Echocardiography in Patients with History of Nonsevere Acute Myocarditis. J Am Soc Echocardiogr 2017;30:756-62.

20. Boussuges A, Rives S, Finance J, Brégeon F. Assessment of diaphragmatic function by ultrasonography: Current approach and perspectives. World J Clin Cases 2020;8:2408-24.

21. Eun LY, Lee YM. Myocardial Layer-Specific Strain Analysis in Children with Mitochondrial Disease. Yonsei Med J 2018;59:128-34.

22. Nemes A, Kalapos A, Domsik P, Orosz A, Lengyel C. Correlations between left ventricular rotational mechanics and parasympathetic autonomic function-results from the three-dimensional speckle-tracking echocardiographic MAGYAR-Healthy Study. Quant Imaging Med Surg 2021;11:1613-8.

23. Xing X, Li D, Chen S, Wang L, Li Z, He L. Evaluation of left ventricular systolic function in patients with different 
types of ischemic heart disease by two-dimensional speckle tracking imaging. J Cardiothorac Surg 2020;15:325.

24. Cong J, Lee Y, Fu X, Wang Z, Wang W, Lee J. Quantitative evaluation of longitudinal strain in layerspecific myocardium in patients with preeclampsia. Int $\mathrm{J}$ Cardiovasc Imaging 2018;34:193-200.

25. Triposkiadis F, Butler J, Abboud FM, Armstrong PW, Adamopoulos S, Atherton JJ, et al. The continuous heart failure spectrum: moving beyond an ejection fraction classification. Eur Heart J 2019;40:2155-63.

26. Xu L, Wang N, Chen X, Liang Y, Zhou H, Yan J. Quantitative evaluation of myocardial layer-specific strain using two-dimensional speckle tracking echocardiography among young adults with essential hypertension in China. Medicine (Baltimore) 2018;97:e12448.

27. Sun M, Dong Y, Wang Y, Li G, Huang D. Assessment of the left ventricular function in patients with uremia using layer-specific 2-dimensional speckle tracking echocardiography. Medicine (Baltimore) 2019;98:e14656.

28. Bakkestrøm R, Christensen NL, Wolsk E, Banke A, Dahl JS, Andersen MJ, Gustafsson F, Hassager C, Møller JE. Layer-specific deformation analysis in severe aortic valve stenosis, primary mitral valve regurgitation, and healthy individuals validated against invasive hemodynamic measurements of heart function. Echocardiography 2018;35:170-8.

29. Addetia K, Takeuchi M, Maffessanti F, Nagata Y, Hamilton J, Mor-Avi V, Lang RM. Simultaneous Longitudinal Strain in All 4 Cardiac Chambers: A Novel Method for Comprehensive Functional Assessment of the Heart. Circ Cardiovasc Imaging 2016;9:e003895.

30. Yu H, Tang D, Geva T, Yang C, Wu Z, Rathod RH, Huang X, Billiar KL, Del Nido PJ. Ventricle stress/strain comparisons between Tetralogy of Fallot patients and healthy using models with different zero-load diastole and systole morphologies. PLoS One 2019;14:e0220328.

Cite this article as: Chen Z, Zhang S, Fang A, Shao J, Shen H, Sun B, Guo G, Liu L. Early changes in left ventricular myocardial function by $2 \mathrm{D}$ speckle tracking layer-specific technique in neonates with hyperbilirubinemia. Quant Imaging Med Surg 2022;12(1):796-809. doi: 10.21037/qims-21-197
31. Yaman B, Akpınar O, Kemal HS, Cerit L, Sezenöz B, Açıkgöz E, Duygu H. The beneficial effect of lowintensity exercise on cardiac performance assessed by two-dimensional speckle tracking echocardiography. Echocardiography 2020;37:1989-99.

32. Jiang L, Wang J, Liu X, Li ZL, Xia CC, Xie LJ, Gao Y, Shen MT, Han PL, Guo YK, Yang ZG. The combined effects of cardiac geometry, microcirculation, and tissue characteristics on cardiac systolic and diastolic function in subclinical diabetes mellitus-related cardiomyopathy. Int J Cardiol 2020;320:112-8.

33. Pinto SI, Campos JB. Numerical study of wall shear stressbased descriptors in the human left coronary artery. Comput Methods Biomech Biomed Engin 2016;19:1443-55.

34. Badano LP, Addetia K, Pontone G, Torlasco C, Lang RM, Parati G, Muraru D. Advanced imaging of right ventricular anatomy and function. Heart 2020;106:1469-76.

35. Qiao YY, Zeng M, Li RJ, Leng ZT, Yang J, Yang Y. Layerspecific myocardial strain analysis: investigation of regional deformation in a rabbit model of diabetes mellitus during different stages. Med Ultrason 2016;18:339-44.

36. Cantinotti M, Scalese M, Giordano R, Franchi E, Assanta N, Marotta M, Viacava C, Molinaro S, Iervasi G, Santoro G, Koestenberger M. Normative Data for Left and Right Ventricular Systolic Strain in Healthy Caucasian Italian Children by Two-Dimensional Speckle-Tracking Echocardiography. J Am Soc Echocardiogr 2018;31:712720.e6.

37. Verdugo-Marchese M, Coiro S, Selton-Suty C, Kobayashi M, Bozec E, Lamiral Z, Venner C, Zannad F, Rossignol P, Girerd N, Huttin O. Left ventricular myocardial deformation pattern, mechanical dispersion, and their relation with electrocardiogram markers in the large population-based STANISLAS cohort: insights into electromechanical coupling. Eur Heart J Cardiovasc Imaging 2020;21:1237-45. 